\title{
Thematic relatedness production norms for 100 object concepts
}

\author{
Olessia Jouravlev $^{1,2} \cdot$ Ken McRae $^{1}$
}

Published online: 28 October 2015

(C) Psychonomic Society, Inc. 2015

\begin{abstract}
Knowledge of thematic relations is an area of increased interest in semantic memory research because it is crucial to many cognitive processes. One methodological issue that researchers face is how to identify pairs of thematically related concepts that are well-established in semantic memory for most people. In this article, we review existing methods of assessing thematic relatedness and provide thematic relatedness production norming data for 100 object concepts. In addition, 1,174 related concept pairs obtained from the production norms were classified as reflecting one of the five subtypes of relations: attributive, argument, coordinate, locative, and temporal. The database and methodology will be useful for researchers interested in the effects of thematic knowledge on language processing, analogical reasoning, similarity judgments, and memory. These data will also benefit researchers interested in investigating potential processing differences among the five types of semantic relations.
\end{abstract}

Keywords Thematic relations $\cdot$ Concepts $\cdot$ Semantic memory $\cdot$ Production norms

Most of our prior knowledge about the nature of thematic processing has come from studies in which researchers have directly compared thematic and taxonomic forms of organization

Electronic supplementary material The online version of this article (doi:10.3758/s13428-015-0679-8) contains supplementary material, which is available to authorized users.

Olessia Jouravlev

olessiaj@mit.edu

1 University of Western Ontario, London, Ontario, Canada

2 McGovern Institute for Brain Research, Massachusetts Institute of Technology, Cambridge, MA 02139, USA of concepts in semantic memory. Taxonomic relations are based on the comparison of features of concepts (Hampton, 2006; Markman, 1989), with concepts that have many features in common often being taxonomically related. Thematic relations, on the other hand, are defined as those that are built around a certain theme, event, or scenario (Estes, Golonka, \& Jones, 2011). The general consensus is that taxonomic and thematic relations rely on two functionally distinct, but complementary, modes of conceptual organization in semantic memory. The dissociation of taxonomic versus thematic processing has been documented in behavioral (Borghi \& Caramelli, 2003; Lin \& Murphy, 2001; Masuda \& Nisbett, 2001; Mirman \& Graziano, 2012), neuroimaging (Kalénine, Mirman, \& Buxbaum, 2012; Sachs, Weis, Krings, Huber, \& Kircher, 2008; Schwartz et al., 2011), and patient (Davidoff \& Roberson, 2004) studies. Furthermore, thematic processing has been shown to be as crucial in language comprehension, analogy making, similarity judgment, and memory processes as is taxonomic knowledge (for a review, see Estes et al., 2011). Considering how important thematic knowledge is to many cognitive processes, it is not surprising that thematic thinking is an area of increased interest in semantic memory research.

One of the methodological challenges for a researcher examining thematic relations is that of selecting thematically related concept pairs. This is not an easy task, because thematic relations are often subjective in a way that individuals might have different conceptual knowledge of events and situations. For example, the concepts "dog" and "park" might have a strong thematic link in memory for dog owners who walk their dogs every morning in a park, but not so much for people who do not have pets. Furthermore, people can imagine a common event or scenario for almost any two concepts, and thus integrate these two concepts thematically. For instance, even if one has never experienced the concepts "dog" and "ice cream" within the same event or scenario, one might be able 
to draw a thematic link between such concepts. Thus, thematic links are somewhat subjective and can be established between any two concepts referring to objects that afford interactions.

Researchers deal with these methodological issues by focusing on conventional thematic relations, defined as relations that have salient, well-established representations in the semantic memory of most people (Estes et al., 2011). The logical question, however, is how conventional and nonconventional thematic relations can be differentiated. In theory, conceptual relations become conventionalized due to the frequent co-occurrence of corresponding objects in real-life situations and in linguistic descriptions of them. Thus, the frequency of exposure to the same combination of objects within the same event is what determines the strength of the thematic relations between concepts in semantic memory and, hence, the degree of conventionality of a particular thematic relationship.

In practice, this methodological issue has been addressed in a number of ways in prior studies of thematic processing. First, some researchers do not elaborate on the methodology of stimulus selection, which presumably indicates that they relied solely on their own intuition in deciding what concepts are linked by conventional thematic relations (Bonthoux \& Kalenine, 2007; de Zubicaray, Hansen, \& McMahon, 2013; Kalénine et al., 2009). Other researchers have used a relation verification task to select their materials. In some cases, participants classified pairs of concepts as being taxonomically related, thematically related, or unrelated (Kriukova, Bridger, \& Mecklinger, 2013; Maguire, Brier, \& Ferree, 2010). In others, participants rated on a 7-point Likert scale the extent to which each pair is linked together in a common scenario or in a functional relationship (Jones \& Golonka, 2012; Kalénine et al., 2012; Kircher, Sass, Sachs, \& Krach, 2009). A slightly different validation approach was taken by Lin and Murphy (2001), who asked participants to write a sentence describing how two given concepts go together to form a category, and, then, on the basis of these responses, the authors categorized the pairs of concepts as thematic or taxonomic.

These previously used methods of assessing the conventionality of thematic relations undoubtedly have a number of advantages, with the ease and speed of identifying the strengths of thematic links being just a couple of the practical benefits. On the other hand, these methods are not without some limitations. For example, relation verification tasks based on classifying concept pairs as thematic, taxonomic, or unrelated can provide rough estimates of the strengths of the thematic links between concepts. Pairs of concepts that are consistently categorized as thematic are presumably strongly thematically related. However, variation in the degree of conventionality of thematic relations across items classified as being thematically related cannot be established with this task. Tasks using thematic relatedness rating scales, on the other hand, do capture differences in conventionality, but such ratings are relative to the other items in a list. When participants are presented with a relatively short list of items consisting of equal proportions of thematically related, taxonomically related, and unrelated pairs of concepts, their ratings tend to be influenced by the other items in the list. For example, "blouse-hanger" might be rated as more conventional than it really is due to the fact that its thematic relatedness is established relative to "blouse-cherry" (unrelated) or "blouse-shirt" (taxonomic), which appear in the same list.

Thematic relatedness production norms are an alternative method for estimating the strength of thematic relatedness and conventionality of concept pairs. In this type of norming, a large number of participants can be asked to produce words corresponding to concepts that they consider to be thematically related to the presented cue word. Although collecting and scoring production norms is a lengthy and effortful process, researchers might acquire more representative data than those obtained using rating or categorization methods. First, production norms provide an aggregate of a large number of responses tapping into the semantic memory of many people. Thus, thematic production norms are likely to uncover concept pairs that are conventional and generalizable to the population. The second benefit is the improved scope of the representation of conceptual memory space that production norms can provide. Indeed, the results of a relatedness rating study can inform researchers only about the relationships existing in selected concept pairs. For example, on the basis of the relatedness rating results for the pair "cat-milk," a researcher can conclude only that these particular concepts are strongly related. The data obtained in the production task, on the other hand, go far beyond that. Since participants are free to produce any thematically related response to a given cue concept (e.g., "cat"), researchers might learn how many and what other concepts are linked thematically to the given concept (e.g., "milk," "mouse," "couch," "fish," "tree," etc.), and how strong these links are in relation to each other. Thus, the data obtained in the production norms can provide a better approximation of the organization of networks of thematic connections for a given concept than do data from relatedness rating norms.

Thematic production norms share some features with word association norms (Fernandez, Diez, Alonso, \& Beato, 2004; Nelson, McEvoy, \& Schreiber, 2004). In particular, both provide measures of the production frequency of a particular word as a thematically related or associated response to another word. Production frequency is interpreted as an index of the strength of the semantic connection between two concepts in both types of norms. At the same time, thematic production norms and word association norms differ in their purposes. In particular, the instructions make the thematic production norms more specifically targeted at types of thematic relations than at the general semantic, and possibly nonsemantic (i.e., phonologically or orthographically based), associations that the word association norms provide. Furthermore, due to the 
fact that word association norm instructions usually limit participants to only one response for each cued word, it is possible that some semantic (including thematic) relations that are likely to be in people's memory would not be included in association norms. Indeed, a priming effect for thematically, but not associatively, related prime-target pairs (according to Nelson et al.'s, 2004, norms) has been demonstrated (Perraudin \& Mounoud, 2009). This evidence suggests that free association norms do not provide a complete description of the thematic relations existing in people's memory. Therefore, the collection of thematic relatedness production norms that specifically target this type of relation in semantic memory is an important task. In a number of prior studies examining thematic thinking (Hare, Jones, Thomson, Kelly, \& McRae, 2009; McRae, Hare, Elman, \& Ferretti, 2005), the researchers selected their stimuli on the basis of production norms. However, a limited number of items and/or types of thematic relations were examined in those prior studies.

The aim of the present research was to create freely available thematic relatedness production norms, and to serve as an exemplar of this methodology. In the first part of our norming study, thematically related word responses to 100 cue words (otherwise unconstrained) were collected. The frequencies of different responses cued by the same word were computed on the basis of the data of 100 participants and were taken to reflect the strength of thematic connections with a cue word. Thus, for each cue word, a network of concepts linked to it by thematic links of different strengths was identified. This network provides an approximation of the thematic relations existing in semantic memory. The thematic relatedness norms can help researchers in selecting stimuli for studies examining the effects of thematic knowledge on cognition (e.g., language processing, memory, analogy making, etc.) or studies investigating differences in thematic versus taxonomic processing.

In the second part of the study, participants classified pairs of related concepts obtained from the production norms as reflecting one of the following subtypes of semantic relations: attributive (e.g., "baker-apron"), argument (e.g., "bearfish"), coordinate (e.g., "beer-chips"), locative (e.g., "cheese-pizza"), temporal (e.g., "church-Sunday"), or other. The proposed classification is not exhaustive and does not include all possible types of thematic relations identified in prior research (Borghi \& Caramelli, 2003; Estes, Golonka, \& Jones, 2011). Furthermore, currently there is not general agreement about a comprehensive typology of thematic relations (for a discussion, see McRae, Khalkhali, \& Hare, 2012). It is not clear right now how many types of thematic relations there are, what these relations are, and whether there are processing differences among them. Nevertheless, existing behavioral (Hare et al., 2009; Muehlhaus et al., 2013), electroencephalographic, and neuroimaging (Muehlhaus et al., 2014) evidence suggests that subtypes of thematic relations are, indeed, distinct, as is indicated by the differential patterns of responses evoked by different types of thematic relations. Comparative examination of the nature of subtypes of thematic relations and their underlying processing mechanisms is currently an important research question in semantic relatedness research. Therefore, norms even for some, but not all, types of thematic relations provided in the current research would be useful for researchers addressing the issue of the nonuniformity of subtypes of thematic relations.

Out of all possible thematic relations, we decided to focus on the five subtypes (attributive, argument, coordinate, locative, and temporal) for a number of reasons. First, in most of the prior research, investigators have predominantly used items that exemplified one of these five types of relationships. For example, in the study of thematic processing by Lin and Murphy (2001), $40 \%$ of the thematically related items were in locative, $21 \%$ were in argument, $18 \%$ were in attributive, $11 \%$ were in coordinate, and $5 \%$ were in temporal relations. In the study by Jones and Golonka (2012), $19 \%$ of the thematically related items were locative, $11 \%$ were attributive, $8 \%$ were argument, $7 \%$ were temporal, and $6 \%$ were coordinate relations. ${ }^{1}$ Furthermore, having conducted a preliminary examination of the production norms obtained in Study 1 , we identified that these five types of thematic relations appeared to be the most common types in our database of thematically related pairs of words. The data obtained in our second study will be useful to researchers who wish to investigate potential processing differences between the subtypes of thematic relations.

\section{Study 1: Thematic relatedness production norms}

\section{Method}

Subjects A total of 200 students from the University of Western Ontario (age: $M=19, S D=2 ; 135$ females) participated for course credit. All of the participants indicated that English was their native language.

Materials and procedures The stimuli were 100 concrete concepts, most of which have been used in previous studies of thematic relations (Estes \& Jones, 2009; Golonka \& Estes, 2009; Lin \& Murphy, 2001). The stimuli were randomly distributed over two lists of 50 words. Each list was presented to 100 participants in a production norming study conducted online. First, participants were provided with the definition of a thematic relationship (see Supplemental Material, Appendix A for the instructions). Then, they were asked to

\footnotetext{
${ }^{1}$ A classification of the thematically related items used in Lin and Murphy (2001) and Jones and Golonka (2012) according to the subtypes of relations was conducted post-hoc by the first author and, thus, provides only a rough estimation of the distribution of types of thematic relations in their stimuli.
} 
read the words referring to concrete concepts and to write down at least three names of other living or nonliving things that might interact with them or be related to them. The instructions stated that participants should come up with words that correspond to nouns. Participants were also advised against writing down the names of objects that are taxonomically related to each cue word.

In a number of prior studies, researchers have reported the existence of an implicit bias toward taxonomic responses, which is formed, allegedly, as the result of formal education during which the taxonomic representation of knowledge is favored. To minimize this bias, we instructed participants to avoid providing taxonomic responses. A major goal was to avoid the production of superordinate (e.g., "cat-animal") and subordinate (e.g., "cat-Siamese") categories. These concept pairs clearly are not thematically related. The main issue concerns category coordinates (i.e., similar concepts); some similar concepts do not participate in thematic relations, and our goal was to minimize this type of response. For example, we did not want participants to generate "jet" and "helicopter," given the cue "airplane." On the other hand, some similar concepts do participate in thematic relations, such as "cat" and "dog." Because we included examples of similar concepts in our instructions as a type of response to avoid, thematically related pairs that are also taxonomically related might be underrepresented in our production norms. On the other hand, "dog" and "mouse" were the top two responses to "cat," and the norms include many such examples. Therefore, it appears that participants did indeed produce similar concepts that also participate in thematic relations.

\section{Results and discussion}

The list of cue words and the participants' responses are provided online as Supplementary Material A. For each cue word, the number of people who provided the same response word was computed. In addition, the number of responses weighted by the order in which responses were given was calculated. Thus, the responses given first were multiplied by 3 , the responses given second were multiplied by 2 , and all other responses were multiplied by 1 .

The first author coded participants' responses in such a way that words referring to the same concept were grouped together. In such cases, the counts for the less frequently mentioned words were added to the counts of the most frequently mentioned (and otherwise synonymous) word. For example, in response to the word "airplane," 11 participants produced "flight attendant," and two produced "stewardess." These counts were added together and coded under the most frequent response, "flight attendant." Decisions about synonymy were based on information from the www.thesaurus.com and www. dictionary.reference.com sites, which list words' synonyms.

Participants' responses were diverse. On average, 64 unique responses $(S D=11)$ were produced per cue. There was a great deal of variability in the production frequencies of the responses to cue words across participants. Production frequency can be considered as a measure of conventionality, meaning that frequently produced pairs are present in semantic memory for many people. Most of the responses were produced by a single participant or by a few participants only (i.e., these responses were highly idiosyncratic). For example, the following responses to the cue "dog" were provided by only one out of 100 participants tested: "squirrel," "shepherd, " "shoe," "vet," "hole," "snail," "bell," "coat," "paper," "mailman," "harness," "garbage," "fish," and "fun." Although it is possible to link these concepts within a common scenario (e.g., Pavlov's dogs responding to the sound of a bell), it is likely that the conventionality of these relations is very low in the population. At the same time, for each target word, some concept names appeared repeatedly in participants' responses. As in many other production studies that have used a subjective criterion to identify the most typical responses (McRae, Cree, Seidenberg, \& McNorgan, 2005; Nelson et al., 2004), we decided to examine further only responses that were produced by at least ten out of 100 norming participants. In all, 1,174 responses met this criterion, with an average of 12 conventional responses $(S D=3)$ being produced per cue. For instance, the cue "dog" evoked the following responses in at least ten out of 100 participants: "leash," "cat," "bone," "ball," "park," "house," "kennel," "treat," "dog food," "collar," "fur," "toy," and "tail." The assumption is that these conventional pairs of concepts have strong connections in the semantic memory of many people.

As we discussed in the introduction, the greatest advantage of the production norms is that they provide researchers not only with information about the strength of a thematic connection between two given concepts, but also about the relative rankings of the connections in the conceptual space of a given item. For example, the strengths of the links in the pairs of concepts "dog-leash" and "mechanic-tools" are very similar (.39, meaning that 39 out of 100 people gave this response, vs. .40). If we had ratings for only these two pairs, we would expect to see no processing differences for these two items (assuming that all other lexical characteristics known to impact the processing of words were controlled for). However, if we considered the relative rankings of the responses, we would note that the response "leash" to the cue "dog" is the most frequent one, whereas the response "tools" to the cue "mechanic" is ranked second (the "mechanic" cue evoked the response "car" most frequently, .76). Hypothetically, this difference in the rankings of the relatedness of a pair could have a major impact on the performance in various tasks. For example, any model based on the principles of interactive activation and competition (McClelland \& Rumelhart, 1981), such as the associative read-out model (Hofmann, Kuchinke, Biemann, Tamm, \& Jacobs, 2011), would predict that the strongest thematic link should be activated more quickly than a thematic 
link of the same nominal but not the same relative strength (i.e., it is the second, third, or fourth most strongly related concept), due to the presence of strong competition from thematic links with higher ranks, in the latter case.

Similarly, one might expect that the processing of a particular thematic link would depend on the number of other links present in the same conceptual space (i.e., the thematic neighborhood). The more "neighbors" are in the neighborhood, the greater the competition among them should be. This increased competition for selection in dense thematic neighborhoods might, hypothetically, slow down performance. Thus, one might predict that people would be slower to process thematic links in the conceptual space of "dog," which includes 13 concepts linked to it, than to process thematic relations in the conceptual space of "vase," which has only four concepts linked to it ("flower" $=.86$, "water" $=.63$, "glass" $=.40$, and "table" = .19).

Of course, empirical investigations of the effects of the density of thematic neighborhoods and the relative strength of each connection on the processing of thematically related words will be needed before any conclusions about the roles of these factors in thematic thinking can be drawn. The information about the number of concepts in a thematic neighborhood and their relative strengths, which may be obtained in production but not in rating-based norms and which we provide in the present research for 100 concepts, may be useful for future examinations of factors that may impact the speed of thematic processing.

To conclude, the norms obtained in our production studies provide an approximation of the thematic networks for each concept. A thematic network is a network of conventional thematic relations that exist between a given concept (e.g., "dog") and a set of other concepts thematically linked to it (e.g., "cat," "bone," "leash," etc.). An illustration of the representation of the thematic network for the concept "dog" is provided in Fig. 1.

\section{Study 2: Types of relation rating norms}

\section{Method}

Subjects A total of 500 students of the University of Western Ontario (age: $M=18.86$ years, $S D=2.15 ; 313$ females, 187 males) received course credit for their participation. All of the participants identified English as their native language.

Materials and procedures Although the participants in Study 1 were asked to provide noun responses only, and to avoid producing the names of taxonomically related things, some participants did not follow these instructions. Therefore, the data were further reduced by removing the responses that

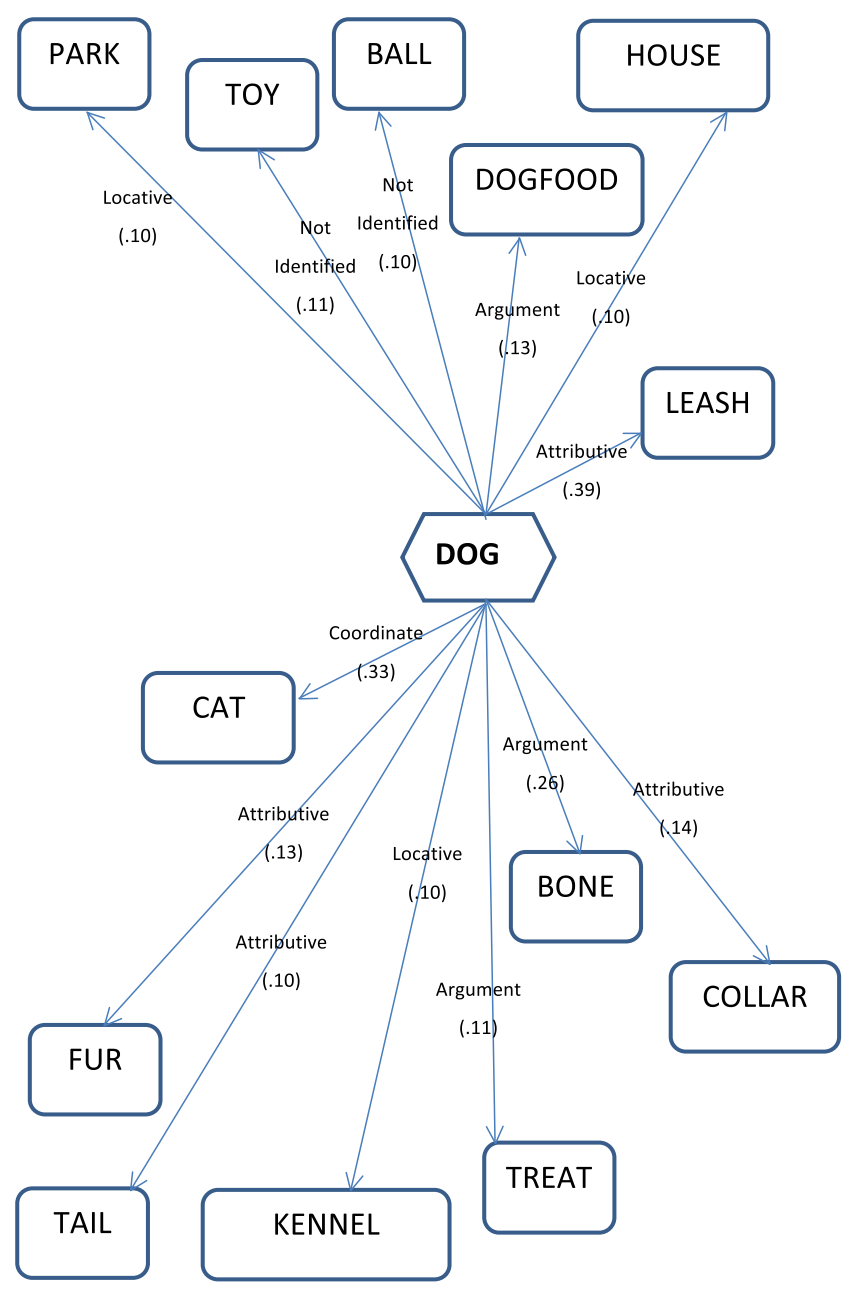

Fig. 1 Representation of the thematic network for the concept "dog." The length of a connection corresponds to the conventionality of the relationship between two concepts (based on the results of Study 1). The frequency of each response to the cue word "dog" is given in parentheses. The indicated types of thematic relationships are based on the results of Study 2

corresponded to adjectives referring to some quality of a target (e.g., "mouse-small") and to verbs referring to some action of a target (e.g., "dog-barking"). We also removed responses corresponding to nouns referring to superordinate or subordinate concepts that are related to the target concepts (e.g., "horse-animal," "shoes-sneakers"). Although the instructions for Study 1 discouraged participants from producing taxonomically related responses, this type of response was still provided to some extent. Some participants responded to cues with the names of more general (superordinate-level) or more specific (subordinate-level) concepts. Superordinate and subordinate relations are clearly taxonomic and were eliminated from the database because the concept pairs do not participate in thematic relations. On the other hand, taxonomic relations at the same level of categorization refer to pairs of concepts that may also be thematically related (e.g., "dog-cat," "forkknife"). Therefore, taxonomically related items at the same 
level of categorization were retained in the database and used in Study 2. Finally, in Study 1, we had combined synonymous responses to cue words (e.g., the responses "stewardess" and "flight attendant" to the cue word "airplane" were combined). The rationale was that thematic relations are primarily conceptual rather than lexical. By combining responses that correspond to different lexical items but to the same concept, we were able to provide a better approximation of the conceptual representations of thematic relationships. In addition, in the database of thematically related cue-response pairs that we obtained in Study 1, in a few instances a synonymous to a cue word response was provided (e.g., "rabbit-bunny"). Because these clearly do not correspond to thematic relations, such pairs were eliminated. As a result, our database of related pairs of concepts that we used to identify different types of thematic relations across pairs of concepts included 1,025 items.

Concept pairs were randomly distributed over ten testing lists, with 102 or 103 pair per list. Each list was presented to 50 participants in a norming study conducted online. First, the participants read definitions of the five relationships: attributive, argument, coordinate, locative, and temporal (see Supplementary Material, Appendix B for the instructions). Attributive relations were defined as relations between an object and one of its characteristics or properties (e.g., "bakerapron"). Argument relations are relations between an object that performs some action causing changes in the state of another object (e.g., "bear-fish"). Coordinate relations are those between two objects that happen to be part of the same action/event, but that do not interact with each other directly (e.g., "beer-chips"). Locative relations exist between an object and its typical location (e.g., "doctor-hospital"). Finally, temporal relations are those between an object and a point in time (e.g., "church-Sunday").

To test whether participants understood the types of thematic relations, they were asked to explain to the experimenter what each type of thematic relation means. All participants defined the five relations successfully. Therefore, no participant was excluded from the main part of the relatedness rating study. Participants read pairs of related words and decided on the type of relationship that was the most suitable description of an interaction/relationship between two concepts. Participants were also provided with an option to indicate that none of the five relationships was appropriate.

\section{Results and discussion}

The complete list of pairs of words and participants' responses is provided online as a Supplemental Material B. For each item, we counted the number of times that each thematic relationship was selected by participants. We assumed that there was a general agreement on the type of relationship that characterizes an interaction occurring between two concepts if at least 25 out of 50 participants selected it. For 779 of the items
(76 \%), there was a consensus. Attributive (302 pairs) and locative (216 pairs) relations were the most frequent ones in our database. They were followed by argument (133 pairs), coordinate ( 90 pairs), and temporal (35 pairs) relations. This predominance of attributive and locative relations might be reflective of the special role that object identity and object location play in human cognition (Kohler, Moscovitch, \& Melo, 2001; Milner \& Goodale, 2008).

Interestingly, we observed some noticeable differences in the distributions of types of relations as a function of the semantic category to which a cue concept belonged. For example, for concepts belonging to the category buildings/ organizations (e.g., "school," "bank," "church," and "hospital"), attributive relations were the most common $[M($ cases per concept $)=3.18]$, followed by locative $[M($ cases per concept $)=0.36]$, argument $[M$ (cases per concept $)=0.27]$, coordinate $[M($ cases per concept $)=0.09]$, and temporal $[M$ (cases per concept $)=0.09]$. This distribution suggests that for concepts belonging to the category "buildings/ organizations," the identification of the type of building/organization, based on its observable attributes, is more crucial than, for example, knowledge of the location of a building/ organization or the times when it is attended.

For the category people (e.g., "teacher," "postman," "baker," and "woman"), attributive relations were also the most common $[M$ (cases per concept $)=4.27]$. Furthermore, the thematic networks of concepts belonging to the category "people" were also rich in argument $[M$ (cases per concept $)=$ 2.46], locative $[M$ (cases per concept $)=1.45]$, and coordinate $[M$ (cases per concept $)=0.91]$ relations. Thus, for concepts belonging to the category "people," not only is the identification of a person (or his/her profession) central in the semantic memory space, but also the knowledge of what kind of objects/people can be changed as a result of the actions of this person (e.g., a mechanic will fix a car, a postman will deliver a letter, or a baby will eat milk), what the most common locations for this person are, and the other types of people with which this person interacts.

For concepts belonging to the categories animals (e.g., "bear," "cat," and "monkey") and foods (e.g., "beer," "cake, " and "ice cream"), the most common type of relationship was locative [animals: $M$ (cases per concept) $=3.5$; foods: $M$ (cases per concept $)=4.0]$. This is not surprising if we consider the importance of knowledge of the locations of objects belonging to these two categories for human survival. Similarly, it is central for our survival to be able to identify foods and animals correctly. Therefore, in our semantic memory, knowledge about the attributes of foods and animals is of quite high importance, as is reflected in the high numbers of attributive relations produced in response to the cue concepts belonging to these two categories [animals: $M$ (cases per concept $)=2.67$; foods: $M$ (cases per concept) $=3.0]$. For concepts in the category animals, thematically related concepts in argument 
$[M($ cases per concept $)=2.06]$, and coordinate $[M$ (cases per concept $)=1.00]$ relations were also produced frequently by participants. Thus, information about the types of objects that might be changed as a result of animals' actions and about types of animals that live in proximity to each other occupies a key place in our semantic memory. For concepts in the category foods, coordinate $[M$ (cases per concept $)=1.50]$ and temporal $[M$ (cases per concept $)=1.0]$ relations were common. Indeed, it is useful to know what food items go well together (i.e., coordinate) and when certain foods are to be consumed.

The final category of concepts that we will touch upon briefly are instruments (e.g., "knife," "scissors," and "hammer"). The primary goal of any instrument is to change the state of certain objects. This is nicely reflected in the dominance of argument relations in the thematic networks of concepts belonging to this category $[M$ (cases per concept $)=$ 4.00]. Because completing a task often requires a person to use a combination of instruments, to select the right instrument for the job, and to find each instrument at a certain location, it is not surprising that coordinate $[M$ (cases per concept $)=2.75]$, attributive $[M$ (cases per concept $)=2.25]$, and locative $[M$ (cases per concept $)=2.25]$ relations are also strongly represented in the thematic networks of concepts referring to instruments.

This brief review of the distributions of five types of thematic relations across five categories clearly shows that different types of thematic relations are not represented equally across different categories of concepts. We posit that the centrality of certain thematic relation for a category is reflective of the types of knowledge about the objects of a category and their interactions with other objects that are essential for human survival (e.g., knowledge of location for food concepts, but knowledge of the objects to which an item should be applied, for instrument concepts). This idea that types of thematic relations play differential roles in processing items from particular categories is supported by existing empirical research. Tsagkaridis, Watson, Jax, and Buxbaum (2014) demonstrated that when processing manipulable artifact concepts (e.g., "wine bottle"), participants preferred action-based thematic relations (e.g., "corkscrew"; referred to as argument relations in the present research) to thematic relations in which the objects do not directly interact with one another (e.g., "cheese"; referred to as coordinate relations in the present research). Whether the centrality of certain types of thematic relations has an impact on human processing of a wide range of categories remains to be examined (e.g., is it easier for people to process concepts in locative relations if those concepts are foods or animals than if they are instruments?).

For pairs of concepts that were categorized as reflecting one of the examined types of thematic relations, there was an overall high consistency in selecting this particular type of relation ( $73 \%$ agreement rate across participants). More detailed information about the distribution of thematic responses as a function of the dominant relationship is provided in Table 1. At the same time, for 246 pairs of words there was no general agreement among participants on the most likely type of thematic relation that exists for a given pair of concepts. For instance, "spider-web" was classified as an argument relation by 24 participants (i.e., a spider weaving the web), but as a locative relation by 16 participants (i.e., a spider being located on the web). Pairs of concepts like "spider-web, " for which no dominant thematic relation was identified, can be thought of as being ambiguous from the point of view of their thematic relatedness. Does this ambiguity in thematic connections cause any processing costs for such items? This is another potential research question that might be addressed using the database we provide.

\section{Conclusion}

The goal of this research was to provide researchers investigating the organization of semantic memory and the processing of

Table 1 Distribution of types of relationship responses $(\max =50)$ as a function of the dominant relationship (in bold)

\begin{tabular}{|c|c|c|c|c|c|c|c|}
\hline \multirow[t]{2}{*}{ Dominant Relationship } & \multirow[t]{2}{*}{ Number of Cases } & \multicolumn{6}{|c|}{ Number of Responses $($ maximum $=50$ ) } \\
\hline & & Coordinate & Locative & Argument & Attributive & Temporal & Other \\
\hline Coordinate & 90 & 35.65 & 2.80 & 4.45 & 3.23 & 0.75 & 3.12 \\
\hline Locative & 216 & 3.04 & 38.42 & 3.00 & 3.17 & 0.81 & 1.57 \\
\hline Argument & 133 & 4.94 & 2.82 & 32.80 & 5.98 & 0.98 & 2.48 \\
\hline Attributive & 302 & 4.03 & 2.94 & 4.29 & 36.05 & 0.69 & 1.99 \\
\hline Temporal & 35 & 2.85 & 3.71 & 2.48 & 1.48 & 38.30 & 1.18 \\
\hline Other & 3 & 5.00 & 6.33 & 7.32 & 3.00 & 1.67 & 26.68 \\
\hline Unidentified & 246 & 9.74 & 8.78 & 11.65 & 10.97 & 1.46 & 7.40 \\
\hline Mean & & 9.32 & 9.40 & 9.43 & 9.12 & 6.38 & 6.35 \\
\hline
\end{tabular}

For a given pair of words, the dominant relationship was identified as such if at least 25 out of 50 participants provided a corresponding thematic relationship response. Number of cases refers to the count of the pairs of words, out of the 1,025 tested, that were characterized as having a specific thematic relationship. 
thematically related concepts, in particular, with a database of normed pairs of thematically related concepts. This database provides estimates of the strength of thematic relatedness for a large number of concept pairs, and hence it informs researchers about the degree of thematic conventionality of each pair. These norms were collected for 100 concrete nouns. At least three thematically related word responses for each cued word were collected from 100 participants. The data obtained are likely to reflect the complex network of thematic links between concepts in semantic memory. Furthermore, production frequency provides an estimate of how strongly various concepts are connected to each other. In Study 2, pairs of related concepts were classified by participants as one of the following subtypes: attributive, argument, coordinate, locative, temporal, or other.

Our norms were collected using a sample of undergraduate students receiving education in Canada. Although we believe that these norms should generalize to similar populations, we are also aware that individual and cultural differences exist in the ways that thematic knowledge is organized. As has been noted, thematic relations are established through experiences that people have with different objects and entities within certain events. Undoubtedly, these experiences may differ across cultures. For example, Canadians are most likely to see monkeys in zoos, whereas in India, monkeys may be seen in other contexts. These differences in experiences with monkeys across cultures might be reflected in differences in the organization of thematic networks for the concept "monkey" in these populations. Differences in the organization of thematic knowledge might be observed even within the same culture. For instance, there might be generational differences in the thematic networks of concepts such as "computer" and "cell phone." Collecting thematic production norms in divergent populations in order to examine potential differences in the organizations of thematic knowledge across cultures and sets of individuals would be an interesting future step. The data of the two norming studies provided here will be of benefit to researchers interested in examining the impact of thematic knowledge on human cognition, differences between thematic versus taxonomic thinking, and potential processing differences for various types of semantic knowledge.

Author note This work was supported by Natural Sciences and Engineering Council Grant Number OGP0155704 to K.M.

\section{References}

Bonthoux, F., \& Kalenine, S. (2007). Preschoolers' superordinate taxonomic categorization as a function of individual processing of visual vs. contextual/functional information and object domain. Cognition, Brain, Behavior, 11, 713-731.

Borghi, A. M., \& Caramelli, N. (2003). Situation bounded conceptual organization in children: From action to spatial relations. Cognitive Development, 18, 49-60.
Davidoff, J., \& Roberson, D. (2004). Preserved thematic and impaired taxonomic categorisation: A case study. Language and Cognitive Processes, 19, 137-174.

de Zubicaray, G. I., Hansen, S., \& McMahon, K. L. (2013). Differential processing of thematic and categorical conceptual relations in spoken word production. Journal of Experimental Psychology: General, 142, 131-142.

Estes, Z., Golonka, S., \& Jones, L. L. (2011). Thematic thinking: The apprehension and consequences of thematic relations. In B. H. Ross (Ed.), The psychology of learning and motivation (Vol. 54, pp. 249294). Burlington, VT: Academic Press.

Estes, Z., \& Jones, L. L. (2009). Integrative priming occurs rapidly and uncontrollably during lexical processing. Journal of Experimental Psychology: General, 138, 112-130. doi:10.1037/a0014677

Fernandez, A., Diez, E., Alonso, M. A., \& Beato, M. S. (2004). Freeassociation norms for the Spanish names of the Snodgrass and Vanderwart pictures. Behavior Research Methods, Instruments, \& Computers, 36, 577-584. doi:10.3758/BF03195604

Golonka, S., \& Estes, Z. (2009). Thematic relations affect similarity via commonalities. Journal of Experimental Psychology: Learning, Memory, and Cognition, 35, 1454-1464.

Hampton, J. A. (2006). Concepts as prototypes. In B. H. Ross (Ed.), The psychology of learning and motivation: Advances in research and theory (Vol. 46, pp. 79-113). San Diego, CA: Elsevier Academic Press.

Hare, M., Jones, M., Thomson, C., Kelly, S., \& McRae, K. (2009). Activating event knowledge. Cognition, 111, 151-167.

Hofmann, M. J., Kuchinke, L., Biemann, C., Tamm, S., \& Jacobs, A. M. (2011). Remembering words in context as predicted by an associative read-out model. Frontiers in Psychology, 2, 252. doi:10.3389/ fpsyg.2011.00252

Jones, L. L., \& Golonka, S. (2012). Different influences on lexical priming for integrative, thematic, and taxonomic relations. Frontiers in Human Neuroscience, 6, 205. doi:10.3389/fnhum.2012.00205

Kalénine, S., Mirman, D., \& Buxbaum, L. J. (2012). A combination of thematic and similarity-based semantic processes confers resistance to deficit following left hemisphere stroke. Frontiers in Human Neuroscience, 6, 106. doi:10.3389/fnhum.2012.00106

Kalénine, S., Peyrin, C., Pichat, C., Segebarth, C., Bonthoux, F., \& Baciu, M. (2009). The sensory-motor specificity of taxonomic and thematic conceptual relations: A behavioral and fMRI study. NeuroImage, 44, 1152-1162.

Kircher, T., Sass, K., Sachs, O., \& Krach, S. (2009). Priming words with pictures: Neural correlates of semantic associations in a cross-modal priming task using fMRI. Human Brain Mapping, 30, 4116-4128. doi: $10.1002 / \mathrm{hbm} .20833$

Köhler, S., Moscovitch, M., \& Melo, B. (2001). Episodic memory for object location versus episodic memory for object identity: Do they rely on distinct encoding processes? Memory \& Cognition, 29, 948 959. doi:10.3758/BF03195757

Kriukova, O., Bridger, E., \& Mecklinger, A. (2013). Semantic relations differentially impact associative recognition memory: Electrophysiological evidence. Brain and Cognition, 83, 93-103.

Lin, E. L., \& Murphy, G. L. (2001). Thematic relations in adults' concepts. Journal of Experimental Psychology: General, 130, 3-28. doi:10.1037/0096-3445.130.1.3

Maguire, M., Brier, M., \& Ferree, T. (2010). EEG theta and alpha responses reveal qualitative differences in processing taxonomic versus thematic semantic relationships. Brain and Language, $114,16-25$.

Markman, E. M. (1989). Categorization and naming in children: Problems of induction. Cambridge, MA: MIT Press.

Masuda, T., \& Nisbett, R. E. (2001). Attending holistically versus analytically: Comparing the context sensitivity of Japanese and Americans. Journal of Personality and Social Psychology, 81, 922-934. 
McClelland, J. L., \& Rumelhart, D. E. (1981). An interactive activation model of context effects in letter perception: I. An account of basic findings. Psychological Review, 88, 375-407. doi:10.1037/0033295X.88.5.375

McRae, K., Cree, G. S., Seidenberg, M. S., \& McNorgan, C. (2005a). Semantic feature production norms for a large set of living and nonliving things. Behavior Research Methods, 37, 547-559. doi: 10.3758/BF03192726

McRae, K., Hare, M., Elman, J. L., \& Ferretti, T. (2005b). A basis for generating expectancies for verbs from nouns. Memory \& Cognition, 33, 1174-1184. doi:10.3758/BF03193221

McRae, K., Khalkhali, S., \& Hare, M. (2012). Semantic and associative relations in adolescents and young adults: Examining a tenuous dichotomy. In V. F. Reyna (Ed.), The adolescent brain: Learning, reasoning, and decision making (pp. 39-66). Washington, DC: American Psychological Association.

Milner, A. D., \& Goodale, M. A. (2008). Two visual systems re-viewed. Neuropsychologia, 46, 774-785. doi:10.1016/j.neuropsychologia. 2007.10.005

Mirman, D., \& Graziano, K. M. (2012). Individual differences in the strength of taxonomic versus thematic relations. Journal of Experimental Psychology: General, 141, 601-609. doi:10.1037/ a0026451

Muehlhaus, J., Heim, S., Altenbach, F., Chatterjee, A., Habel, U., \& Sass, K. (2014). Deeper insights into semantic relations: An fMRI study of part-whole and functional associations. Brain and Language, 129, 30-42. doi:10.1016/j.bandl.2014.01.003

Muehlhaus, J., Heim, S., Sachs, O., Schneider, F., Habel, U., \& Sass, K. (2013). Is the motor or the garage more important to the car? the difference between semantic associations in single word and sentence production. Journal of Psycholinguistic Research, 42, 37-49.

Nelson, D. L., McEvoy, C. L., \& Schreiber, T. A. (2004). The University of South Florida free association, rhyme, and word fragment norms. Behavior Research Methods, Instruments, \& Computers, 36, 402 407. doi:10.3758/BF03195588

Perraudin, S., \& Mounoud, P. (2009). Contribution of the priming paradigm to the understanding of the conceptual developmental shift from 5 to 9 years of age. Developmental Science, 12, 956-977.

Sachs, O., Weis, S., Krings, T., Huber, W., \& Kircher, T. (2008). Categorical and thematic knowledge representation in the brain: Neural correlates of taxonomic and thematic conceptual relations. Neuropsychologia, 46, 409-418.

Schwartz, M. F., Kimberg, D. Y., Walker, G. M., Brecher, A., Faseyitan, O. K., Dell, G. S., \& Coslett, H. B. (2011). Neuroanatomical dissociation for taxonomic and thematic knowledge in the human brain. Proceedings of the National Academy of Sciences, 108, 8520-8524. doi:10.1073/pnas.1014935108

Tsagkaridis, K., Watson, C. E., Jax, S. A., \& Buxbaum, L. J. (2014). The role of action representations in thematic object relations. Frontiers in Human Neuroscience, 8, 140. 